\title{
Einleitung
}

Kaum ein Philosoph hat eine ähnliche Wende in der Entwicklung seines Denkens genommen wie Ludwig Wittgenstein. Wenngleich die Arbeitsbereiche der Früh- und Spätphilosophie zumindest unter der gemeinsamen Bezeichnung Sprachphilosophie geführt werden dürften, könnten die jeweiligen Ausprägungen im Detail jedoch nicht unterschiedlicher ausfallen. Beiden Phasen gemein ist, dass ihre Ergebnisse und Werke über die Grenzen der Philosophie hinaus gewirkt haben. Insbesondere gilt dies für den Begriff der Familienähnlichkeit und dessen Auswirkungen auf die Entwicklung der Prototypensemantik oder die offensichtlichen Paralellen zwischen Formalismus und Funktionalismus in der Linguistik einerseits sowie den entsprechenden Sprachkonzeptionen Wittgensteins im Tractatus und den Philosophischen Untersuchungen andererseits.

Dieser interdisziplinäre Austausch ist bisher jedoch ein relativ einseitiger, wobei die Linguistik Konzepte Wittgensteins aufgriff - nicht jedoch umgekehrt. Die Entwicklungen der funktionalen und kognitiven Linguistik der letzten Jahrzehnte haben jedoch Erkenntnisse zum Verständnis, der Produktion und dem Erlernen von Sprache hervorgebracht, die auch (kontroverse) Kernthemen Wittgensteins betreffen und den Austausch in umgekehrter Richtung motivieren können. Genau dies soll mit dieser Arbeit geschehen, allen voran in Bezug auf den Begriff der Grammatik und deren Willkür. Die Wahl dieses Themas motiviert sich nicht zuletzt aus den eben genannten Gründen: So ermöglicht die Linguistik mit Universalienforschung, Ikonizität und Grammatikalisierung genauere Einblicke in menschliche Sprachverarbeitungsmechanismen und das Funktionieren von Grammatik, wohingegen Wittgensteins Ausführungen zu diesen Themen mitunter ein unheitliches Bild abgeben und zeitweise recht spekulativ erscheinen können. Im Idealfall würden mit den Mitteln und Ergebnissen der linguistischen Forschung also die Thesen Wittgensteins erhellt und darüber hinaus auch diskutiert und überprüft werden können.

Dreh- und Angelpunkt einer solchen Diskussion soll hier nun die Abkehr Wittgensteins von der logisch orientierten Frühphilosophie des Tractatus sein, welche in der Forschung für gewöhnlich als die Wende zu einem sprachlichen Relativismus porträtiert wird, der, dem Diktum des „Bedeutung als Gebrauch" folgend, Tür und Tor für willkürliche Begriffsbildung öffnet. Für eine solch lockere Beziehung zwischen Welt und Sprache spricht sicherlich die Vielfalt der existierenden Sprachen, dagegen jedoch deren Ähnlichkeiten und die Möglichkeit der Übersetzung. Dass es Wittgenstein 
jedoch um viel mehr geht, als um (normal-)sprachliche Variation, zeigt sich schon an seinem eigentümlichen Grammatik-Begriff.

Wittgensteins Überlegungen zu Grammatik und deren Willkürlichkeit nehmen eine zentrale Position in seinem Spätwerk ein. Die Auseinandersetzung damit in der Forschung ist bisher jedoch eher spärlich. Dies mag unter anderem daran liegen, dass Wittgensteins Gedanken zu diesen Themen nicht vollständig in dem Werk seines späteren Wirkens ausgearbeitet sind, welches er für eine Publikation aufgearbeitet hat, nämlich den Philosophischen Untersuchungen. Die Ausführungen zu Grammatik und deren Willkür finden sich stattdessen verstreut in Werken wie Philosophische Grammatik, Bemerkungen über die Grundlagen der Mathematik, Über Gewißheit und Zettel, deren Status im Gesamtwerk ein geringerer oder zumindest weniger offizieller ist. Zudem neigen Wittgensteins Ansichten bei dem Thema grammatischer Willkür noch stärker dazu, ein uneinheitliches Bild abzugeben, als es viele andere seiner Themen ohnehin schon tun. Diese Arbeit soll also zunächst dazu dienen, die Ausführungen zu Grammatik und ihrer Willkürlichkeit in einen Bezugsrahmen mit weiteren Kernthemen des Spätwerks zu setzen und alsdann Ambiguitäten und Unklarheiten dieser Konzeptionen zu minimieren.

Der erste Eindruck der oben genannten Texte ist wahrscheinlich, dass Wittgenstein in seinen Betrachtungen zumindest keinen festen Standpunkt annimmt,${ }^{1}$ denn einerseits sagt er, Grammatik sei willkürlich, ${ }^{2}$ andere Stellen wiederum scheinen das Gegenteil zu belegen. ${ }^{3}$ Dieser scheinbare Widerspruch hebt jedoch nur den Anteil von Willkürlichem und Nicht-Willkürlichem in der Grammatik hervor, so dass es in Zettel für einen besonderen Teil unserer Grammatik - das Farbsystem - lautet: „Hat denn dieses System etwas Willkürliches? Ja und nein. Es ist mit Willkürlichem verwandt, und mit Nichtwillkürlichem. " ${ }^{4}$ Folglich wird der erste, exegetische Teil der Arbeit dazu dienen, Wittgensteins Position zur Willkürlichkeit der Grammatik genauer herauszuarbeiten und zu zeigen, was er an ihr als willkürlich betrachtet und was nicht. Aufgrund des angedeuteten fragmentarischen Charakters der Schriften und der Verzahnung des Grammatik-Begriffs mit verschiedenen anderen Themen, wird die Diskussion desselben jeweils auch Bezüge auf immer wieder auftauchende Kernthemen wie das Privatsprachenargument, das Phänomen der Familienähnlichkeit und die Gebrauchstheorie der Bedeutung nehmen. Der zweite Teil wid hingegen dazu dienen, diese Position im Lichte neue-

\footnotetext{
${ }^{1}$ Forster wirft Wittgenstein Unklarheit und Inkonsistenz vor. Vgl. Forster 2004, S. 2

${ }^{2}$ Vgl. PU §497; PG I, §68; Z, §320.

${ }^{3}$ Vgl. Z, §358.

${ }^{4} \mathrm{Z}$, §358. Kursivierung H.V.
} 
rer Forschungsergebnisse aus der Linguistik und anderen Disziplinen zu diskutieren.

Eine wesentliche Grenze grammatischer Willkür stellt für Wittgenstein nun deren menschliche Komponente dar. Auch wenn Wittgenstein detailliertere Ausührungen zu diesem Thema vermissen läßt, so ist doch mit Verweis auf das bekannte Beispiel der Kommunikation mit Löwen offenbar, dass es sich hierbei um die Grenzen beschränkter linguistischer Kapazität des Gehirns, der lautbildenden Organe und des Wahrnehmungsapparates handelt. Dem gegenüber steht für Wittgenstein außerdem die soziale Grenze, welche grammatische Willkür einschränkt. Diese Komponente wird zumindest über die Bemerkungen zum Regelfolgen behandelt. Da Wittgenstein Grammatik-Begriff aber wesentlich weiter gefaßt ist als der alltägliche, soll zunächst für eine Teilmenge des Wittgensteinschen Grammatikbegriffs - nämlich den der Alltagssprache - mit den Erkenntnissen und Methoden der Linguistik überprüft werden, wie sich grammatische Willkür bzw. die Motivation von Zeichen und Strukturen in menschlicher Sprache ausnimmt. Über die Untersuchung dieser Teilmengen sollen deren Ergebnisse dann durch Rückschlüsse über die Struktur der übergeordneten Menge für Wittgenstein fruchtbar gemacht werden. Im Grunde bedeutet dies eine Umkehrung der historischen interdisziplinären Transferrichtung, da bisher vor allem die Linguistik Beispiele und Begriffe Wittgensteins genutzt und ausgebaut hat - allen voran die Ausführungen zur Familienähnlichkeit und die sich schließlich auch in der Linguistik wieder findenden unklaren Grenzen in der Prototypensemantik. ${ }^{5}$ Kann mit der Linguistik nämlich gezeigt werden, dass sich im alltagssprachlichen Bereich durchaus alternative Grammatiken analog dazu finden, wie Wittgenstein dies angedacht hat, so erscheinen die spekulativeren Beispiele weitaus weniger schwach bzw. sogar durchaus folgerichtig.

\section{Aufbau der Arbeit}

Geführt werden soll der Nachweis alternativer Grammatiken in der alltagssprachlichen Teilmenge Wittgensteinscher Grammatik vor allem für die Bereiche, die Wittgenstein selbst für seine Argumente heranzieht, d.h. für Farben, Zahlen, den Spracherwerb und die Übersetzung. Zuvor (Kapitel 1) müssen jedoch Wittgensteins Methodologie und die Begrifflichkeiten der Grammatik geklärt sowie in Relation zu den sie berührenden Aspekten des Privatsprachenargumentes, der Bemerkungen zum Regelfolgen und der Bedeutungstheorie gesetzt werden.

\footnotetext{
${ }^{5}$ Vgl. Heider 1971.
} 
Da sich durch Wittgensteins Theorie alternativer Grammatiken auch Probleme ergeben, die mit der Erkennbarkeit, dem Verständnis und der Übersetzung solcher Alternativen ergeben, wird die Gavagai-Problematik Quines als Prüfstein herangezogen, da sich hier ein Großteil derselben Probleme passgenau in einer alltagssprachlichen oder zumindest alltagssprachlicheren Situation wiederfinden lässt. Die Situation des Forschers in einer bisher unbekannten Sprachgemeinschaft, der mit dem Ausdruck "Gavagai" und einem vorbeihoppelnden Hasen konfrontiert ist und nun zu entscheiden habe, was dieser Ausdruck bedeute, soll in diesem Sinne als Fortführung und Zuspitzung der oben genannten Probleme von Wittgensteins These modelliert werden. Die Diskussion der entsprechenden Positionen Wittgensteins und Quines leistet Kapitel 1.4.

Die darauffolgenden Kapitel diskutieren zentrale Positionen und Methodologien Wittgensteins im Lichte der neueren Forschung, so etwa die Rolle der Metapher, die sich besonders deutlich in der Darstellung von Sprache als Spiel ausdrückt (Kapitel 2), die Mathematik und ihr menschlicher Ursprung (Kapitel 3), sowie den Bereich der Farben und mögliche alternative Grammatiken wie das Rötlichgrün (Kapitel 4).

Kapitel 5 wendet sich von der Diskussion von Einzelbeispielen insofern ab, als dass zwar der menschliche Spracherwerb und damit das näher betrachtet wird, was bei Wittgenstein noch immer als die menschliche Grenze grammatischer Willkür bezeichnet werden könnte. Letztlich muss aber die Frage gestellt werden, ob die von Wittgenstein selbst vorgenommene und von der Wittgenstein-Forschung alsdann übernommene Einteilung in menschliche und soziale Grenzen grammatischer Willkür wirklich so gezogen werden kann und sollte. ${ }^{6}$ Angeborene Neigungen, wie etwa die Zuschreibung eines bisher unbekannten Wortes für ein Objekt in seiner Gesamtheit, geben darüber hinaus starke Gründe gegen die als Abhilfe für die Gavagai-Problematik aufgebrachte radikale Übersetzung Quines.

Kapitel 6 und 7 führen die Ausweitung auf allgemeinere Betrachtungen zur grammatischen Willkür und der Motivation von Zeichen und Strukturen fort. Interjektionen und Onomatopoetika fungieren hierbei als ein erster Hinweis auf Zeichen, die schlichtweg durch Imitation motiviert sind. Weiterhin wird in Kapitel 6 ausführlich das Phänomen der Ikonizität diskutiert, welches motivierte Zeichen weit über den durch Interjektionen und Onomatopoetika abgedeckten Bereich nachzuweisen vermag. Auch dieses Rahmenmodell der Linguistik liefert Argumente gegen die angebliche Unbestimmtheit der Bedeutung der Gavagai-Situation und damit

\footnotetext{
${ }^{6}$ Vgl. zu dieser Einteilung Forster 2005, Kapitel 3.
} 
auch für die nicht besonders stark ausgeführte Position Wittgensteins für die menschliche Grenze grammatischer Willkür.

Der alltägliche bzw. linguistische Grammatik-Begriff gewinnt in Kapitel 7 durch den Prozess der Grammatikalisierung einen diachronen Charakter, der zudem eine Schnittmenge zwischen Lexikon und Grammatik eröffnet. Da es sich hierbei um Prozesse handelt, die zwar durch kognitive Faktoren des Subjekts beeinflusst sind, jedoch nur intersubjektiv auf der Ebene von Sprachgemeinschaften greifen, kann Wittgensteins Position zwar abermals gestärkt werden - die Einteilung in menschliche und soziale Grenzen grammatischer Willkür wird dadurch aber umso fraglicher. Die Schlussbetrachtungen zeigen weitere Forschungsdesiderata auf, insbesondere in Bezug auf den Anspruch an die Korrektheit alltäglicher Sprache bzw. deren Umsetzung in Wittgensteins philosophischem Programm. 
